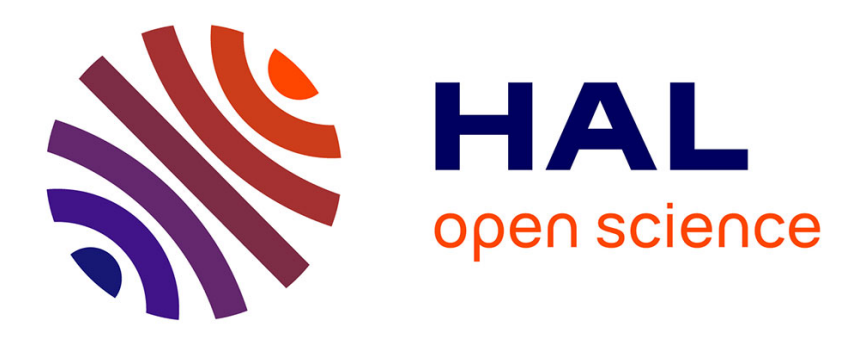

\title{
La plaine de Rivière et le complexe de terrasses et moraines au Sud de Montrejeau
}

Jacques Hubschman

\section{To cite this version:}

Jacques Hubschman. La plaine de Rivière et le complexe de terrasses et moraines au Sud de Montrejeau. Bulletin de l'Association française pour l'étude du quaternaire, 1975, 12 (3-4), pp.192-200. 10.3406/quate.1975.1266 . hal-02736025

\section{HAL Id: hal-02736025 \\ https://hal-univ-tlse2.archives-ouvertes.fr/hal-02736025}

Submitted on 2 Jun 2020

HAL is a multi-disciplinary open access archive for the deposit and dissemination of scientific research documents, whether they are published or not. The documents may come from teaching and research institutions in France or abroad, or from public or private research centers.
L'archive ouverte pluridisciplinaire HAL, est destinée au dépôt et à la diffusion de documents scientifiques de niveau recherche, publiés ou non, émanant des établissements d'enseignement et de recherche français ou étrangers, des laboratoires publics ou privés. 


\section{La plaine de Rivière et le complexe de terrasses et moraines au}

\section{Sud de Montrejeau}

\section{Jacques Hubschman}

\section{Abstract}

Close by the Pyrénées, are laying many fluvial and glacial deposits. Description, analysis of some of there. The discussion concerns chiefly the age of well weathered formations : Mindel or Riss? The problem of the reality of pre-Riss glaciations in the north pyrenean piemont is laid down.

\section{Citer ce document / Cite this document :}

Hubschman Jacques. La plaine de Rivière et le complexe de terrasses et moraines au Sud de Montrejeau. In: Bulletin de I'Association française pour l'étude du quaternaire, vol. 12, n³-4, 1975. pp. 192-200;

doi : https://doi.org/10.3406/quate.1975.1266

https://www.persee.fr/doc/quate_0004-5500_1975_num_12_3_1266

Fichier pdf généré le 19/04/2018 


\title{
La plaine de Rivière et le complexe de terrasses et moraines au sud de Montrejeau
}

\author{
par Jacques HUBSCHMAN
}

\section{SUMMARY}

Close by the Pyrénées, are laying many fluvial and glacial deposits. Description, analysis of some of there. The discussion concerns chiefly the âge of well weathered formations : Mindel or Riss ? The problem of the reality of pre-Riss glaciations in the north pyrenean piemont is laid down.

\section{I. - LES REMBLAIEMENTS DE LA PLAINE DE RIVIERE}

A l'aval de sa confluence avec la Neste, la Garonne s'est ouvert un vaste couloir, souvent large de plus de 4 à $5 \mathrm{~km}$, qui se referme peu à peu à l'approche de Saint-Gaudens (figure 18) : c'est la Plaine de Rivière. Ce couloir vient buter au sud contre les versants de la couverture secondaire plissée nord-pyrénéenne, essentiellement formée de calcaires aptiens et albiens. Au nord, la plaine est dominée par le rebord du Plateau de Lannemezan. Elle est tapissée d'épaisses alluvions dans lesquelles sont taillés plusieurs paliers de terrasses, très faiblement emboîtés les uns dans les autres et souvent discontinus (figure 19). Aux extrémités est et ouest de cet ensemble, la haute terrasse de Montréjeau-SaintGaudens, qui disparaît dans la Plaine de Rivière proprement dite, domine celle-ci par un fort talus de 30 à $40 \mathrm{~m}$ de commandement : on verra que ses caractères

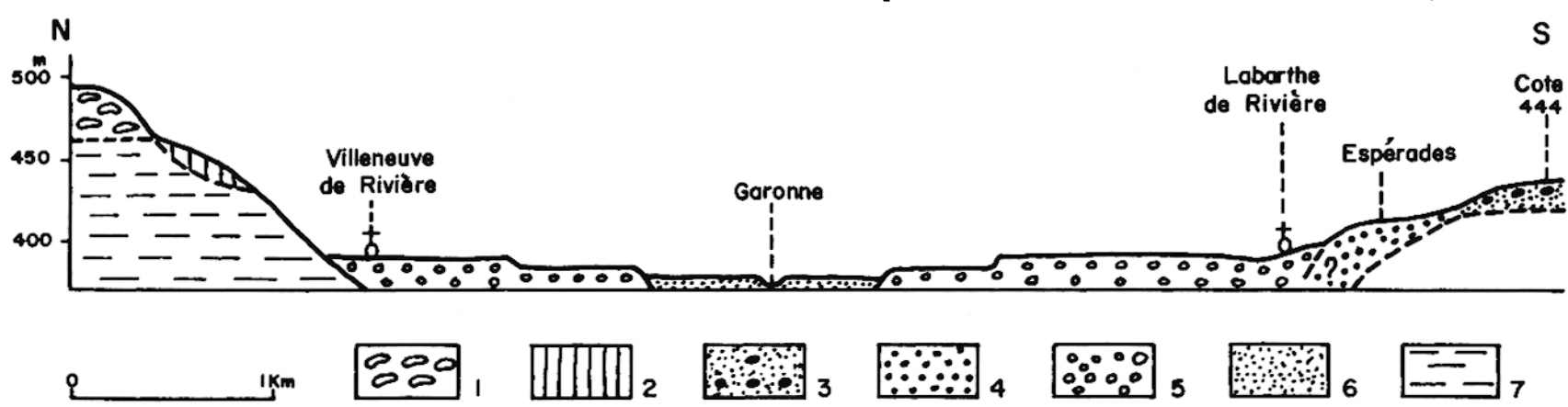

Fig. 19 - La Plaine de Rivière : coupe schématique au droit de Labarthe-de-Rivière.

1. Bordure du plateau de Lannemezan. 2. Replat démantelé dominant Villeneuve-de-Rivière. 3. Lambeau de terrasse de la cote 444. 4. Lambeau de terrasse d'Espéradès. 5. Nappe de la Plaine de Rivière. 6. Lit majeur de la Garonne. 7. Soubassement miocène du plateau de Lannemezan. En blanc, socle calcaire aptien du sud de la Plaine de Rivière. pédo-géochimiques la rattachent au Mindel de l'avantpays molassique (arrêt $n^{\circ} 21$ ). Au sud, plaqués contre ou entre les collines de calcaires urgo-aptiens, subsistent quelques lambeaux de terrasses (figure 19), comme ceux de Labarthe-de-Rivière : Espéradès et cote 444 (arrêt $n^{\circ} 19$ ). Pour ces restes alluviaux et, dans une moindre mesure, pour le remblaiement principal de la Plaine de Rivière, se posent des problèmes d'attribution stratigraphique.

A - Le remblaiement principal de la Plaine de Rivière

L'arrêt $\mathrm{n}^{\circ} 20$ permet d'observer les principaux caractères sédimentologiques du matériel : taille et usure des galets et des blocs, présence d'un manteau limono-caillouteux de 0,5 à $1 \mathrm{~m}$ d'épaisseur recouvrant l'alluvion grossière où les éléments gneisso-granitiques et quartzitiques dominent.

Il faut d'abord, à propos de cette Plaine de Rivière, dissiper une illusion: selon F. Bourdier (communi- 
cation orale), en effet, la glace aurait envahi et recouvert la plaine jusqu'aux abords de Saint-Gaudens. En fait, ce point de vue doit être formellement exclu. D'une part, l'arc morainique de la Serre (figure 20) représente le seul témoignage objectif de l'avancée maximale de la glace (würmienne sans doute, comme on le verra plus loin) dans la cluse du Bazert. D'autre part, le modelé de surface de la Plaine de Rivière est incontestablement celui d'un secteur d'épandage proglaciaire et n'évoque en aucune manière un territoire qui aurait été recouvert par la glace.

Cela dit, le problème qui se pose maintenant est le suivant : le remblaiement de la Plaine de Rivière est-il formé de deux nappes d'âge distinct (Riss et Würm par exemple), comme pourraient le faire penser les petits talus ou "glacis" plus ou moins discontinus qui parcourent la plaine et délimitent grossièrement çà et là deux ou plusieurs paliers? Ou bien, au contraire, s'agit-il d'une seule et même génération alluviale dans laquelle les eaux proglaciaires issues de la cluse du Bazert auraient taillé de loin en loin et notamment de part et d'autre du fleuve, des ébauches de niveaux de terrasses?

C'est cette dernière interprétation qui semble la bonne. En effet, tous les niveaux de la Plaine de Rivière (le lit majeur postglaciaire excepté) montrent des types de sols et d'altération analogues, développés dans des matériaux tout à fait comparables à l'échelle de la plaine.

La coupe de Taillebourg (arrêt $n^{\circ} 22$ ) donne un bon exemple des principaux paramètres sédimentologiques et pédo-géochimiques qui caractérisent ces remblaiements.

\section{Description sommaire de la coupe de l'arrêt $n^{\circ} 22$ : \\ Sol brun faiblement lessivé}

- 0-9 cm: $A_{1}, 10$ YR 4/2, structure granulaire à grenue, racines, galets TRA $10 \%$, limite graduelle, ondulée ;

- 9-35 cm: $A_{2}, 7,5$ YR $5 / 4$, structure légèrement massive à tendance polyédrique, racines, quelques galets, limite inférieure graduelle, ondulée ;

- 35-50 cm: $B_{1 t}, 7,5$ YR $4 / 4$ à $5 / 4$, structure massive en mottes, fentes verticales, quelques revêtements argileux, galets, et graviers TRA 10 à $25 \%$, limite inférieure franche, très ondulée ;

- 50-70 cm: IIB $2 \mathrm{t}$, nappe caillouteuse TRA 60 à $80 \%$, longueur moyenne 12 à $17 \mathrm{~cm}$ et quelques gros blocs, très nombreux graviers en poches, matrice 7,5 YR $5 / 6$ à $4 / 4$ avec poches plus ocres 5 YR 5/6, quelques revêtements argileux, fréquents cristallins à fissures rubéfiées ou globalement fragilisés, exceptionnellement arénisés, limite inférieure graduelle, ondulée ;

- 70-110 cm: IIBC, idem mais matrice 7,5 YR 4/4 à 5 YR $4 / 4$ structure particulaire, limite inférieure graduelle ;

- $110-240 \mathrm{~cm}$ : IIC ${ }_{1}$, idem mais gros blocs plus abondants ;

- 240-680 cm: IIC 2,3 etc., idem mais blocs ou galets calcaires par place avec cimentations locales des galets ou graviers par le calcaire.

\begin{tabular}{|c|c|c|c|c|c|c|}
\hline \multirow{2}{*}{\begin{tabular}{c} 
Prof. \\
\cline { 2 - 6 }
\end{tabular}} & \multicolumn{6}{|c|}{ Granulométrie \% } \\
\cline { 2 - 7 } & & \multicolumn{5}{|c|}{$\%$ de TF } \\
\cline { 2 - 7 } & EG & SG & SF & STF & L & A \\
\hline $20-30$ & 20,1 & 24,3 & 23,4 & 17,3 & 12,8 & 18,8 \\
$40-50$ & 18,7 & 30,6 & 14,3 & 11,5 & 18,3 & 23,1 \\
$55-65$ & 23,7 & 31,9 & 17,5 & 10,1 & 19,8 & 18,9 \\
$75-95$ & 33,6 & 70,5 & 8,6 & 4,8 & 8,5 & 6,1 \\
$115-125$ & 40,0 & 60,3 & 60,3 & 7,6 & 10,6 & 4,3 \\
\hline
\end{tabular}

\begin{tabular}{|c|c|c|c|c|r|r|}
\hline \multirow{2}{*}{$\begin{array}{c}\text { Prof. } \\
\mathrm{cm}\end{array}$} & \multicolumn{2}{|c|}{$\mathrm{pH}$} & \multicolumn{4}{|c|}{ Matière organique \% } \\
\cline { 2 - 7 } & eau & $\mathrm{KCl}$ & MO & $\mathrm{C}$ & $\mathrm{N}$ & $\mathrm{C} / \mathrm{N}$ \\
\hline $20-30$ & 5,6 & 4,5 & 2,1 & 1,2 & 0,12 & 9,3 \\
$40-50$ & 5,5 & 4,5 & 1,4 & 0,82 & 0,09 & 8,9 \\
$55-65$ & 5,4 & 4,3 & 1,2 & 0,73 & 0,09 & 7,4 \\
$75-95$ & 5,7 & 4,9 & 0,6 & 0,35 & 0,06 & 5,8 \\
$115-125$ & 5,9 & 5,0 & 0,4 & 0,24 & 0,02 & 10,3 \\
\hline
\end{tabular}




\begin{tabular}{|c|c|c|c|c|c|c|c|c|c|c|}
\hline \multirow[b]{2}{*}{$\begin{array}{c}\text { Prof. } \\
\mathrm{cm}\end{array}$} & \multicolumn{7}{|c|}{ Complexe absorbant meq $\%$} & \multicolumn{3}{|c|}{$\mathrm{Fe} \%$} \\
\hline & $\mathrm{Ca}$ & $\mathrm{K}$ & $\mathrm{Mg}$ & $\mathrm{Na}$ & $\mathrm{S}$ & $\mathrm{T}$ & S/T \% & FT & FL & FL/FT \% \\
\hline $20-30$ & & & & & & & & 2,84 & 1,50 & 52 \\
\hline $40-50$ & & & & & & & & 3,12 & 1,72 & 52 \\
\hline $55-65$ & 0,7 & 0,0 & 0,0 & 0,0 & 0,9 & 11,7 & 7 & 2,23 & 1,95 & 87 \\
\hline $75-95$ & 0,5 & 0,0 & 0,0 & 0,0 & 0,6 & 10,1 & 6 & 4,91 & 1,28 & 26 \\
\hline $115-125$ & 0,4 & 0,0 & 0,0 & 0,0 & 0,5 & 8,0 & 6 & 4,57 & 1,50 & 32 \\
\hline $230-240$ & & & & & & & & 3,15 & 1,97 & 62 \\
\hline
\end{tabular}

\begin{tabular}{|c|c|c|c|c|c|}
\hline $\begin{array}{c}\text { Prof. } \\
\mathrm{cm}\end{array}$ & Illites & Chlorites & Vermiculite & Kaolinites & $\begin{array}{c}\text { Interstratifiés } \\
10-14 \mathrm{C}, 10-14 \mathrm{~V} \\
14 \mathrm{C}-14 \mathrm{~V}\end{array}$ \\
\hline $20-30$ & $\mathrm{~F}$ & $\mathrm{~m}$ & $\mathrm{~m}$ & $\mathrm{~m}$ & $\mathrm{~m}$ \\
$40-50$ & $\mathrm{M}$ & $\mathrm{m}$ & $\mathrm{m}$ & $\mathrm{f}$ & $\mathrm{f}$ \\
$55-65$ & $\mathrm{M}$ & $\mathrm{m}$ & $\mathrm{m}$ & $\mathrm{f}$ \\
$75-95$ & $\mathrm{~F}$ & $\mathrm{f}$ & $\mathrm{m}$ & $\mathrm{f}$ & $\mathrm{m}$ \\
$115-125$ & $\mathrm{M}$ & $\mathrm{M}$ & & & $\mathrm{m}$ \\
\hline
\end{tabular}

- La différenciation du profil reste peu poussée, bien que la coupe de l'arrêt $n^{\circ} 22$ tranche l'un des paliers les plus élevés du remblaiement de la Plaine de Rivière. En règle générale et selon les conditions locales, le degré d'évolution des profils va des sols bruns modaux ou acides aux sols bruns faiblement lessivés, plus rarement aux sols bruns lessivés. Contrairement aux profils développés dans la basse plaine würmienne à l'aval des Petites Pyrénées (cf. arrêt $\mathrm{n}^{\circ} 6$ bis de la première journée), le front d'altération ne se marque généralement pas très clairement ici. Ce fait doit être mis en rapport avec la nature des éléments gneissogranitiques composant l'alluvion de la Plaine de Rivière. Ces éléments sont en effet beaucoup plus hétérogènes, du point de vue de leur degré d'altération, que dans l'avant-pays molassique: partout, quel que soit le palier ou la profondeur de l'alluvion, des éléments gneisso-granitiques fragilisés et teintés en rouille par le fer, sinon en voie d'arénisation, se mêlent en proportion variable aux galets sains. La matrice sableuse reflète cet état de fait, comme le montre la distribution irrégulière dẹs plages ocre et des taux de libération du fer (cf. tableaux d'analyse).

- La mise en place de l'ensemble du remblaiement principal de la Plaine de Rivière doit être attribuée au Würm. D'une part, en effet, ce remblaiement se raccorde, vers l'aval et au-delà de la cluse de SaintMartory-Boussens, à la basse plaine würmienne de la Garonne. D'autre part, la moraine frontale de la Serre (cf. arrêt $\mathrm{n}^{\circ} 24$, étudié un peu plus loin), d'âge würmien, constitue apparemment le point d'ancrage amont de ce remblaiement et offre à peu près les mêmes caractéristiques pédo-géochimiques.

- On notera, en dernier lieu, que la différenciation pédologique des couvertures limono-caillouteuses de la Plaine de Rivière est sensiblement moins poussée que dans la basse plaine würmienne à l'aval des Prépy. rénées. En fait, on retrouve ici l'influence déterminante du matériel parental. C'est que, dans la zone des basses vallées montagnardes, les couvertures meubles superficielles sont plus riches en minéraux primaires (originaires du Flysch cénomanien et des massifs cristallins voisins) que les limons d'origine molassique de l'avantpays nord-pyrénéen (M. Icole 1973). Il en résulte ainsi une plus forte inertie géochimique et pédologique de départ, renforcée par l'action floculante des ions aluminium, abondants ici. Cette inertie tend à ralentir l'évolution au cours de ses premières étapes, comme l'indique la partie gauche du tableau de la page 168.

\section{B - Les lambeaux de terrasse du sud de la Plaine de Rivière}

Sous la ville de Saint-Gaudens (gare) et au sud de Valentine jusqu'à Cier-de-Rivière, quelques éléments résiduels d'un niveau de terrasse se présentent, plaqués pour la plupart contre les versants de calcaire urgoaptien de la couverture secondaire plissée. Ces lambeaux dominent de 10 à $15 \mathrm{~m}$ environ la Plaine de Rivière proprement dite, comme le montre la coupe de l'arrêt $n^{\circ} 19$, à Labarthe-de-Rivière (figure 19).

\section{Description sommaire de la coupe de l'arrêt $n^{\circ} 19$ : \\ Sol lessivé hydromorphe, profil \\ d'altération à tendance fersiallitique}

- 0-40 cm: Ap puis $A_{2}$ (remaniements), 10 YR $4 / 2$ puis 10 YR $5 / 4$ avec traînées $10 \mathrm{YR} 4 / 2$, racines, pores et canaux, structure polyédrique à granulaire plus massive en profondeur, quelques graviers et galets, limite inférieure graduelle, ondulée ;

- 40-85 cm: $A_{2 \mathrm{~g}}$ puis $B_{1 t g}, 10$ YR $5 / 3$ à taches puis plages rouille 7 YR 5/8 plus larges et abondantes 
en profondeur, structure massive sous-structure polyédrique, concrétions et enduits noirs en profondeur, limite inférieure graduelle, ondulée ;

$-85-115 \mathrm{~cm}: B_{2 \operatorname{tg}}$, marmorisé, structure légèrement polyédrique, revêtements argileux, quelques graviers et galets, limite inférieure en poches, assez franche ;

$-115-170 \mathrm{~cm}: \operatorname{IIB}_{3 \mathrm{tg}}$ à $\operatorname{IIBC}_{1 \mathrm{~g}}, 7 \mathrm{YR} 5 / 8$ à 10 YR 6/8, nappe arénacée à galets et graviers TRA 20 à $30 \%$, structure massive, quelques revêtements argileux et enduits noirs, galets gneisso-granitiques arénisés ou altérés mêlés à d'autres beaucoup plus cohérents et presque frais, limite inférieure franche, ondulée ;

$-170-250 \mathrm{~cm}: \mathrm{IIBC}_{2}$, nappe à gros galets et blocs ou graviers TRA $80 \%$, matrice 10 YR $6 / 8$, plus de $50 \%$ des éléments cristallins arénisés ou altérés, plages argileuses des schistes pourris du Flysch, limite inférieure graduelle, indistincte ;

- 250-390 cm: $\mathrm{IIBC}_{3}$, idem avec plus de débris de Flysch et schistes argileux, matrice pius sableuse et plus jeune.

\begin{tabular}{|c|c|c|c|c|c|c|}
\hline \multirow[b]{3}{*}{$\begin{array}{l}\text { Prof. } \\
\mathrm{cm}\end{array}$} & \multicolumn{6}{|c|}{ Granulométrie \% } \\
\hline & \multirow[b]{2}{*}{ EG } & \multicolumn{5}{|c|}{$\%$ de TF } \\
\hline & & SG & $\mathrm{SF}$ & STF & $\mathrm{L}$ & A \\
\hline $25-40$ & 0 & 7,4 & 15,8 & 24,3 & 23,0 & 27,8 \\
\hline $85-115$ & 0 & 8,5 & 17,4 & 19,5 & 20,3 & 33,1 \\
\hline $130-140$ & 14,0 & 25,8 & 34,9 & 9,9 & 4,8 & 23,3 \\
\hline $200-220$ & 49,5 & 29,3 & 40,7 & 7,0 & 3,2 & 18,1 \\
\hline $250-270$ & 61,1 & 34,2 & 33,6 & 6,5 & 6,6 & 17,3 \\
\hline
\end{tabular}

\begin{tabular}{|c|c|c|c|c|c|c|c|c|}
\hline \multirow{2}{*}{$\begin{array}{c}\text { Prof. } \\
\text { cm }\end{array}$} & \multicolumn{2}{|c|}{$\mathrm{pH}$} & \multicolumn{2}{c|}{$\mathrm{P}_{2} \mathrm{O}_{5} \%_{0}$} & \multicolumn{4}{c|}{ Matière organique \% } \\
\cline { 2 - 9 } & eau & $\mathrm{KCl}$ & citrique & Joret & MO & $\mathrm{C}$ & $\mathrm{N}$ & $\mathrm{C} / \mathrm{N}$ \\
\hline $25-40$ & 6,4 & 4,8 & 0,00 & 0,00 & 0,9 & 0,56 & 0,07 & 7,6 \\
$85-115$ & 5,5 & 4,1 & 0,00 & 0,00 & 0,3 & 0,20 & 0,04 & 4,1 \\
$130-140$ & & 4,9 & 0,00 & 0,00 & 0,2 & 0,12 & 0,03 & 3,3 \\
$200-220$ & & 4,9 & 0,00 & 0,00 & & & & \\
$250-270$ & & 4,7 & 0,00 & 0,00 & & & & \\
\hline
\end{tabular}

\begin{tabular}{|c|l|l|l|l|r|r|r|r|r|r|}
\hline \multirow{2}{*}{$\begin{array}{c}\text { Prof. } \\
\mathrm{cm}\end{array}$} & \multicolumn{9}{|c|}{ Complexe absorbant meq \% } & \multicolumn{4}{c|}{ Fe \% } \\
\cline { 2 - 11 } & $\mathrm{Ca}$ & $\mathrm{K}$ & $\mathrm{Mg}$ & $\mathrm{Na}$ & $\mathrm{S}$ & $\mathrm{T}$ & $\mathrm{S} / \mathrm{T} \%$ & $\mathrm{FT}$ & $\mathrm{FL}$ & $\mathrm{FL} / \mathrm{FT} \%$ \\
\hline $25-40$ & 2,7 & 0,02 & 0,00 & 0,12 & 2,9 & 8,5 & 34 & 2,06 & 1,45 & 72 \\
$85-115$ & 0,82 & 0,04 & 0,00 & 0,09 & 1,0 & 10,7 & 9 & 3,57 & 0,89 & 24 \\
$130-140$ & 6,1 & 0,91 & 2,6 & 0,28 & 10,0 & 15,1 & 66 & 4,85 & 0,61 & 12 \\
$200-220$ & & & & & & & & 4,35 & 2,91 & 67 \\
$250-270$ & & & & & & & & 3,92 & 1,68 & 43 \\
\hline
\end{tabular}

\begin{tabular}{|c|c|c|c|c|c|}
\hline $\begin{array}{l}\text { Prof. } \\
\mathrm{cm}\end{array}$ & Illites & $\begin{array}{c}\text { Chlorites } \\
\text { primaires }\end{array}$ & C-V-Al* & Kaolinites & $\begin{array}{c}\text { Interstratifiés } \\
\text { gonflants }\end{array}$ \\
\hline $25-40$ & $\mathrm{M}$ & $\mathrm{f}$ & $\mathrm{F}$ & $\mathrm{f}$ & $\mathrm{m}$ \\
$85-115$ & $\mathrm{M}$ & $\mathrm{m}$ & $\mathrm{F}$ & $\mathrm{m}$ & $\mathrm{m}$ \\
$130-140$ & $\mathrm{~F}$ & $\mathrm{tr}$ & $\mathrm{m}$ & $\mathrm{m}$ & \\
$200-220$ & $\mathrm{M}$ & $\mathrm{m}$ & $\mathrm{M}$ & $\mathrm{m}$ & \\
$250-270$ & $\mathrm{~F}$ & $\mathrm{~m}$ & $\mathrm{~m}$ & \\
\hline
\end{tabular}

(*) C-V-Al : minéraux à comportement de vermiculite ou chlorite-Al, ou d'interstratifiés 10-14 C, 10-14 V, 14 C-14 V. 


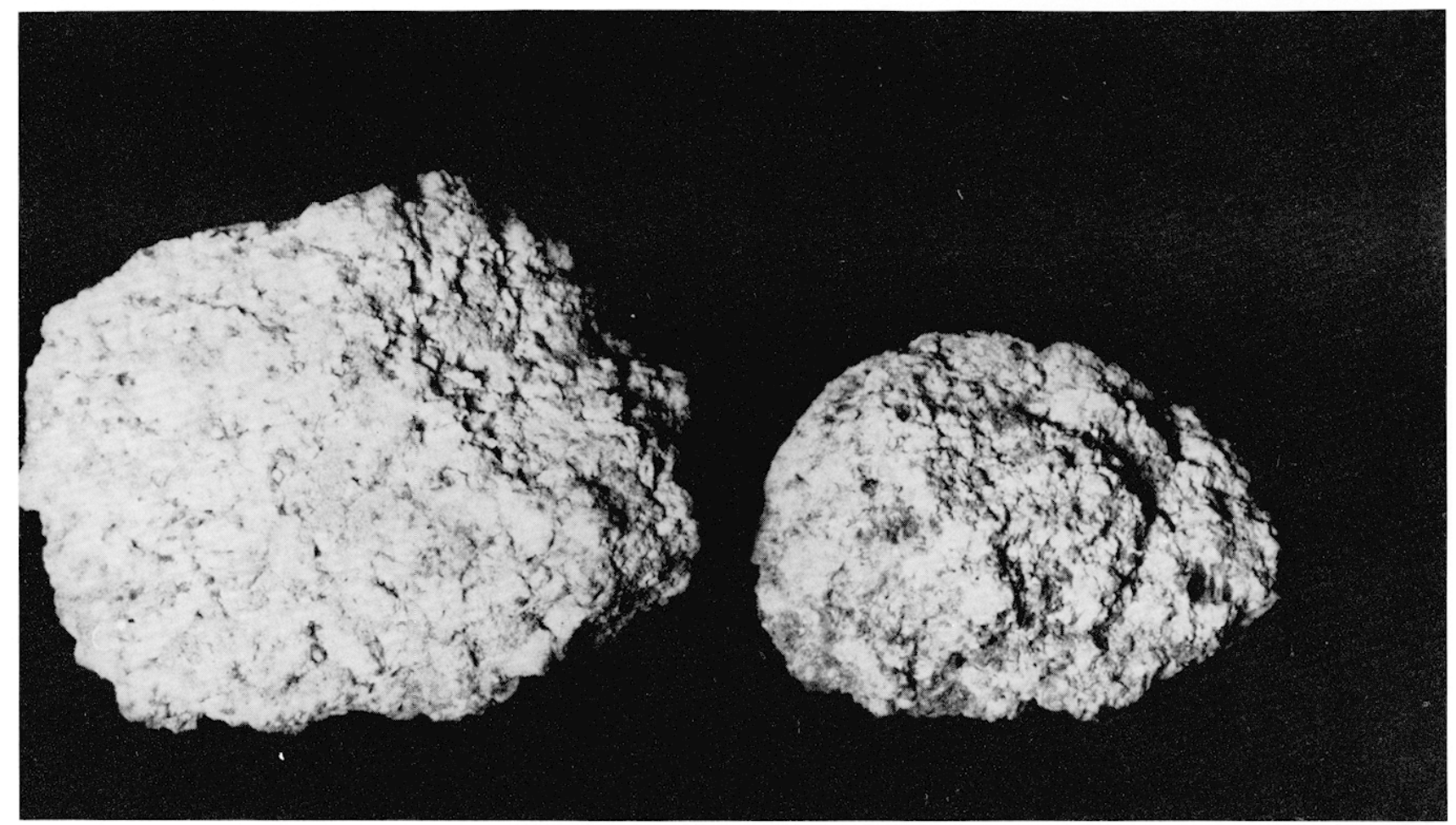

Photo 22 - Coupe de l'arrêt $n^{\circ} 19$. Eléments gneisso-granitiques en voie d'arénisation.

- Le problème posé est celui de l'attribution au Riss ou au Mindel de ce type de matériel.

\section{Les partisans d'une attribution au Mindel mettent} l'accent sur l'altération des matériaux gneissogranitiques, sensiblement plus poussée que dans le Riss des basses terrasses de l'avant-pays, et sur la rubéfaction plus prononcée qui en résulte dans la matrice.

Les partisans d'une attribution au Riss, sans contester le haut degré d'altération atteint par le cailloutis alluvial, s'appuient sur un certain nombre d'arguments (J. Hubschman 1973, 1975a) :

- Bien que le profil de l'arrêt $n^{\circ} 19$ soit manifestement remanié et sans doute tronqué (bordure de terrasse et de vallon), l'altitude relative de ces éléments de terrasse au-dessus du remblaiement principal würmien de la plaine (10 à $15 \mathrm{~m}$ ), comparée à l'ampleur du talus du Mindel qui, à Montréjeau, domine de 30 à $40 \mathrm{~m}$ la Plaine de Rivière, plaide plutôt en faveur du Riss. Mais il est vrai que l'altitude, surtout à proximité de la montagne, n'a guère de signification stratigraphique précise.

- Le mode d'évolution pédo-géochimique de ces cailloutis alluviaux s'apparente plus à celui du Riss, quoiqu'en nettement plus poussé, qu'à celui du Mindel. En particulier, le mélange constant entre éléments gneisso-granitiques arénisés et éléments presque sains ou peu touchés, est tout à fait caractéristique. L'intégration à la matrice des éléments cristallins est moins avancée que dans le Mindel et leur mode d'arénisation rappelle, en plus poussé, celui du Riss (photo 22). L'argilisation des matrices demeure toujours modérée (cf. tableaux d'analyse). Dans ces matrices, les minéraux kaoliniques sont bien mal représentés, en tout cas beaucoup moins que dans le Mindel de Montréjeau (arrêt $n^{\circ} 21$ ). Quant à la rubéfaction de la matrice, elle traduit la forte quoiqu'inégale libération du fer dans un matériel drainé, liée à l'altération avancée de nombreux éléments gneisso-granitiques ou riches en ferromagnésiens.

- Plus poussé que dans le Riss de l'avant-pays mais sensiblement différent de celui du Mindel, ce type d'altération se retrouve dans nombre de basses vallées montagnardes du piémont nord-pyrénéen. Ce faciès traduirait l'abondance initiale, dans les alluvions rissiennes déposées au pied même de la montagne, de matériaux gneisso-granitiques originellement fragiles et rapidement altérables (éventuellement "préparés" pendant la longue phase Mindel-Riss et issus en particulier des massifs primaires nord-pyrénéens). Ainsi s'expliquerait également la part non négligeable d'éléments cristallins en voie d'arénisation dans le Würm (et même le Postglaciaire) de la Plaine de Rivière et aussi dans les moraines würmiennes, comme on le verra un peu plus loin (arrêt $\left.\mathrm{n}^{\circ} 24\right)$.

Une troisième interprétation, proposée par $P$. Barrère, reviendrait à considérer le faciès de l'arrêt $\mathrm{n}^{\circ} 19$ comme les horizons profonds d'une nappe mindélienne originellement beaucoup plus épaisse et fortement décapée par la suite, lors de la fusion des glaces 
rissiennes dont certains lobes diffluents occupaient effectivement les dépressions du massif calcaire urgoaptien, au sud de la Plaine de Rivière. Sans doute l'allure générale du profil et ses paramètres analytiques permettraient-ils d'accepter ce rapprochement. Mais des objections subsistent : où se trouve alors le Riss, dont on ne peut imaginer qu'il ait totalement disparu ? D'autre part, il existe en Ariège nombre de profils identiques, développés dans des nappes autrement mieux conservées.

\section{C-La terrasse mindélienne de Montréjeau-Saint- Gaudens}

L'intérêt majeur de la coupe de l'arrêt $\mathrm{n}^{0} 21$ réside dans le fait que la terrasse de Montréjeau-SaintGaudens représente un bon repère chronostratigraphique local, auquel on pourra comparer les données de l'arrêt $n^{\circ} 19$. Par sa position dans le paysage mais surtout par ses paramètres pédo. géochimiques, cette formation doit être en effet rapportée au Mindel.

L'arrêt $n^{\circ} 21$ concerne une longue tranchée routière qui recoupe localement le Flysch cénomanien, lui aussi altéré.

\section{Description sommaire d'un profil de l'arrêt $n^{\circ} 21$ :}

Sol lessivé hydromorphe à accumulation de fer (grep), profil d'altération à tendance fersiallitique.

$-0-40 \mathrm{~cm}$ : Ap puis $A_{2 g}$ et $B_{1 t g}, 10$ YR 6/3, structure massive à tendance feuilletée puis polyédrique, taches grises et rouille en profondeur avec concrétions et enduits noirs, limite inférieure franche, un peu ondulée ;

$-40-75 \mathrm{~cm}$ : IIB 2 tg , nappe de galets et surtout graviers TRA 60 à $80 \%$, avec niveaux très indurés et cimentés par le fer (grep), très panaché, matrice devenant plus meuble en profondeur, limite inférieure graduelle ;

- 75-180 cm: IIB $_{3 \operatorname{tg}}$ à IIBC $_{1 \mathrm{~g}}$, nappe graveleuse à gros éléments, cristallins altérés et/ou intégrés à la matrice, cortex blancs et rouille des quartzites ou des cristallins, matrice fond 2,5 YR 4/8 à 5 YR $5 / 8$ avec larges bandes subhorizontales très claires 5 YR $6 / 2$ à 5 YR 7/1, nombreux et épais revêtements argileux, limite inférieure graduelle ;

- $180-280 \mathrm{~cm}:$ IIB $_{4 \mathrm{tg}}$ à $\mathrm{IIBC}_{2 \mathrm{~g}}$, idem mais organisation horizontale des bandes de dégradation moins nette, gros galets mieux distribués dans la masse.

\begin{tabular}{|c|c|c|c|c|c|c|}
\hline \multirow{2}{*}{$\begin{array}{c}\text { Prof. } \\
\text { cm }\end{array}$} & \multirow{6}{|c|}{ Granulométrie \% } \\
\cline { 2 - 7 } & EG & \multicolumn{5}{|c|}{$\%$ de TF } \\
\cline { 2 - 7 } & & SG & SF & STF & L & A \\
\hline $15-25$ & 2,5 & 20,3 & 18,8 & 12,8 & 23,7 & 21,9 \\
$40-65$ & - & 21,0 & 16,7 & 11,8 & 19,3 & 29,9 \\
$115-125$ & 34,0 & 32,1 & 17,9 & 5,5 & 11,5 & 31,7 \\
$160-180$ & 23,7 & 28,6 & 15,9 & 3,1 & 10,3 & 40,1 \\
$230-250$ & 42,9 & 31,3 & 13,8 & 4,7 & 12,2 & 35,6 \\
\hline
\end{tabular}

\begin{tabular}{|c|c|c|c|c|c|c|c|c|}
\hline \multirow{2}{*}{$\begin{array}{c}\text { Prof. } \\
\mathrm{cm}\end{array}$} & \multicolumn{2}{|c|}{$\mathrm{pH}$} & \multicolumn{2}{c|}{$\mathrm{P}_{2} \mathrm{O}_{5} \%$} & \multicolumn{4}{c|}{ Matière organique \% } \\
\cline { 2 - 8 } & eau & $\mathrm{KCl}$ & citrique & Joret & MO & $\mathrm{C}$ & $\mathrm{N}$ & $\mathrm{C} / \mathrm{N}$ \\
\hline $15-25$ & 6,9 & 5,9 & 0,02 & 0,00 & 0,9 & 0,54 & 0,08 & 6,7 \\
$40-65$ & 7,2 & 5,9 & 0,01 & 0,00 & 0,4 & 0,25 & 0,06 & 3,8 \\
$115-125$ & 7,1 & 5,8 & 0,00 & 0,00 & 0,2 & 0,14 & 0,05 & 2,6 \\
$160-180$ & 6,9 & 5,3 & & & & & & \\
$230-250$ & 5,4 & 4,5 & & & & & & \\
\hline
\end{tabular}

\begin{tabular}{|c|c|c|c|c|c|c|c|c|c|c|}
\hline \multirow[b]{2}{*}{$\begin{array}{l}\text { Prof. } \\
\mathrm{cm}\end{array}$} & \multicolumn{7}{|c|}{ Complexe absorbant meq $\%$} & \multicolumn{3}{|c|}{$\mathrm{Fe} \%$} \\
\hline & $\mathrm{Ca}$ & $\mathrm{K}$ & $\mathrm{Mg}$ & $\mathrm{Na}$ & $\mathrm{S}$ & $\mathrm{T}$ & $\mathrm{S} / \mathrm{T} \%$ & FT & $\mathrm{FL}$ & $\mathrm{FL} / \mathrm{FT} \%$ \\
\hline $15-25$ & 6,2 & 0,07 & 0,28 & 0,08 & 6,7 & 13,4 & 50 & 1,44 & 0,71 & 49 \\
\hline $40-65$ & 6,4 & 0,06 & 0,25 & 0,09 & 6,9 & 14,1 & 48 & 9,80 & & \\
\hline $115-125$ & 8,6 & 0,06 & 1,0 & 0,09 & 9,8 & 18,5 & 52 & 4,85 (a) & 3,96 & 81 \\
\hline $160-180$ & & & & & & & & 3,77 (a) & 2,80 & 74 \\
\hline $230-250$ & & & & & & & & 3,71 (a) & 2,55 & 69 \\
\hline
\end{tabular}

(a) Matrice à dominante rouge ou ocre. 


\begin{tabular}{|c|c|c|c|c|}
\hline $\begin{array}{c}\text { Prof. } \\
\text { cm }\end{array}$ & Illites & C-V-Al & Kaolinites & $\begin{array}{c}\text { Interstratifiés } \\
\text { gonflants }\end{array}$ \\
\hline $15-25$ & M & M & $\mathrm{f}$ & tr \\
$40-65$ (grep) & M & m & M & f \\
$115-125$ & F & f & M & f \\
$160-180$ & F & m & m & f \\
\hline
\end{tabular}

- Les caractères pédo-géochimiques de ce type de profil sont indiscutablement à attribuer au Mindel classique du piémont garonnais. Par nombre de paramètres (argilisation, intégration à la matrice, kaolinisation...), ils contrastent singulièrement avec les coupes analogues à celle de l'arrêt $\mathrm{n}^{\circ} 19$.

- Les caractères sédimentologiques (gros galets et localement blocs avec éléments de dimension plus réduite) ne permettent pas de trancher en faveur d'une mise en place véritablement fluvio-glaciaire (comme l'admettait H. Alimen, 1964) ou, au contraire, simplement fluviatile. La proximité des bassins d'alimentation montagnards peut en effet parfaitement expliquer le faciès hétérométrique et la présence de blocs importants dans cette nappe mindélienne. On touche ici au problème, depuis longtemps discuté, des glaciations prérissiennes dans les Pyrénées, problème sur lequel on reviendra un peu plus loin.

\section{II. - LE COMPLEXE GLACIAIRE ET FLUVIO-GLACIAIRE DU SUD DE MONTREJEAU}

A - La cluse de Tibiran-Jaunac et la terrasse de Seilhan

A l'entrée de la Plaine de Rivière et en amont de sa confluence avec la Neste, la Garonne a taillé une double cluse dont elle n'emprunte aujourd'hui que la branche occidentale : la cluse de Tibiran-Jaunac (figure 20). L'excellente conservation du vallum morainique würmien de la Serre témoigne que le fleuve n'a abandonné l'autre branche, la cluse de LabroquèreBazert, qu'à une époque récente, vers la fin du Würm. Dans la cluse de Tibiran-Jaunac, en revanche, la Garonne a détruit toute trace de construction morainique würmienne typique. Par contre, un certain nombre de dépôts ont subsisté, sous forme d'éléments de terrasse plus ou moins démantelés. Le meilleur exemple en est donné par la haute terrasse de SeilhanLuc (figure 20). qui, vers $480 \mathrm{~m}$, s'appuie contre la colline de Gourdan.

L'arrêt $n^{\circ} 23$ consiste précisément en une coupe dans la terrasse de Seilhan-Luc. Dans cette coupe, de nombreux éléments gneisso-granitiques sont arénisés ou fortement altérés .(les poches argileuses étant dues à l'altération du Flysch cénomanien argileux) et la matrice sableuse est assez rubéfiée mais fort pauvre en kaolinites. Ce profil pose les mêmes problèmes que celui de l'arrêt $\mathrm{n}^{\circ} 19$ : Riss ou Mindel ?

La question est d'importance car, ici, la réponse qui lui est donnée met directement en jeu l'existence de glaciations prérissiennes et notamment mindélienne. En effet, par sa situation, sa forme et son matériel, la terrasse de Seilhan semble s'apparenter plutôt à une terrasse de marge glaciaire. D'autre part, cette terrasse semble en relation avec les blocs erratiques et pavements cristallins plus ou moins altérés qui, à plus de $150 \mathrm{~m}$ au-dessus de la terrasse, parsèment les collines de Gourdan et Burs. Ces éléments indiquent sans nul doute une phase glaciaire au cours de laquelle l'épaisseur de la glace était bien supérieure à celle des langues

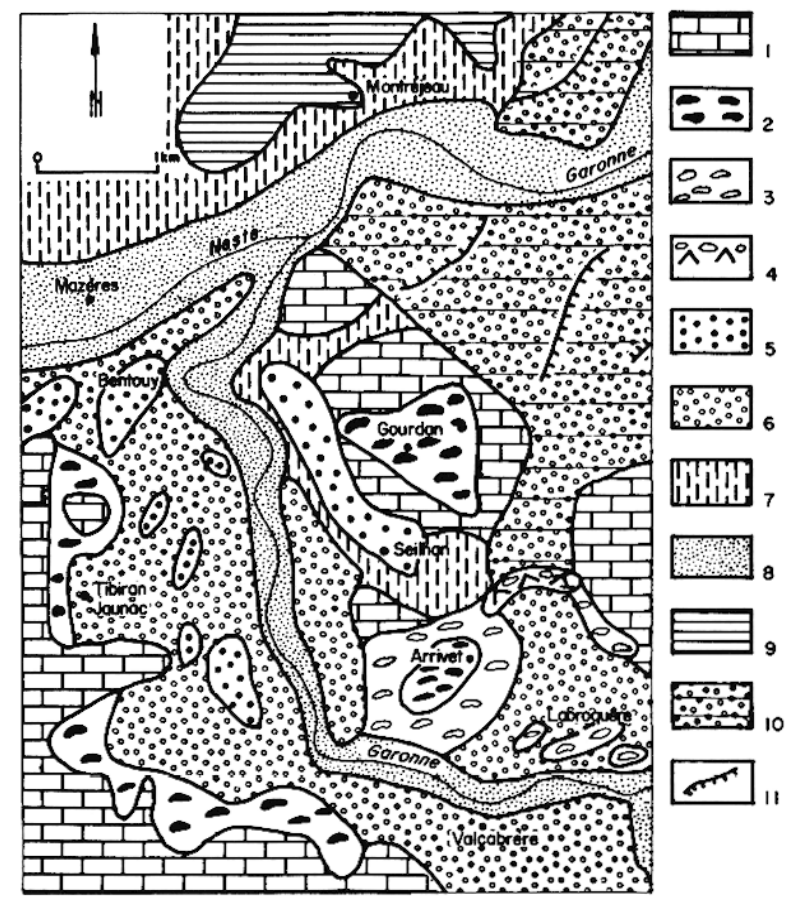

Fig. 20 - Terrasses et moraines garonnaises au sud de Montréjeau (en partie d'après la carte géomorphologique détaillée de la France, feuille Montréjeau sud-ouest, CNRS 1970). 1. Couverture secondaire plissée urgo-aptienne. 2. Placages morainiques anciens discontinus. 3. Dépôts morainiques récents. 4. Vallum de la Serre. 5. Hautes terrasses de Seilhan, Bentouy et niveaux homologues. 6. Nappe fluvio-glaciaire principale de Tibiran-Jaunac et du bassin de Barbazan. 7. Matériel limoneux et limono-caillouteux glissé des talus de terrasses et des pieds de versants. 8. Formations limoneuses et limonocaillouteuses du fond du bassin de Barbazan et du lit majeur de la Garonne. 9. Haute terrasse de Montréjeau-Saint-Gaudens. 10. Remblaiement alluvial de la Plaine de Rivière. 11. Talus des paliers dans le remblaiement alluvial de la Plaine de Rivière. 
würmiennes, quoique, saignées par les diffluences et soumises aux températures déjà plus élevées de ces basses vallées $(400-450 \mathrm{~m})$, les langues glaciaires ne devaient guère venir mourir plus loin qu'à l'entrée de la Plaine de Rivière (F. Taillefer 1969).

Ce "Maximum glaciaire" (P. Barrère 1953, F. Taillefer 1969), probablement responsable de la mise en place de la terrasse de Seilhan, sera donc attribué soit plutôt au Mindel, soit (pour notre part) plutôt au Riss, selon les mêmes arguments que ceux utilisés au cours de la discussion engagée lors de l'arrêt $n^{\circ} 19$. Cependant, la coupe de l'arrêt $\mathrm{n}^{\circ} 24$ évoquée cidessous permettra de saisir avec quelle prudence le degré d'altération des galets cristallins doit être utilisé comme indicateur stratigraphique dans ces basses vallées montagnardes.

\section{B - Les moraines würmiennes de la cluse de Labroquère-Bazert}

$\mathrm{Au}$ centre de la cluse de Labroquère-Bazert, le vallum terminal würmien de la Serre, bel arc morainique culminant vers $485 \mathrm{~m}$, ferme au nord le bassin glaciaire de Barbazan, d'où émergent les quelques lambeaux morainiques de Labroquère (figure 20). Au pic de la colline de Camaroux-Carmont et en prolongement de la branche orientale du vallum de la Serre, les basses pentes sont recouvertes d'un épais matériel glaciaire. La coupe de l'arrêt $n^{\circ} 24$ montre le faciès général de l'alluvion: sol brun-acide superficiel, développé dans un cailloutis hétérométrique à gangue de tendance sableuse, de couleur brun-jaune assez terne. L'état du matériel caillouteux retient particulièrement l'attention. Les galets gneisso-granitiques, abondants, témoignent en effet d'un degré d'altération extrêmement variable : aux éléments pratiquement arénisés se mêlent des galets parfaitement sains et durs, avec tous les stades intermédiaires entre ces deux faciès extrêmes. Il s'agit ici, rappelons-le, d'un dépôt d'âge würmien. Il est vrai que, même dans les galets arénisés, le fer commence à peine à s'extraire des structures cristallines (biotites mordorées et exsudations ferrugineuses localisées), ce qui explique l'absence de rubéfaction de la matrice. Mais le processus de décomposition des galets et des minéraux ferromagnésiens, point de départ de la ferruginisation de la matrice, est déjà bien engagé. On peut donc parfaitement concevoir que le même type de matériaux, déposés au Riss dans ces basses vallées montagnardes, aient pu atteindre rapidement un stade d'évolution poussé (rubéfaction, arénisation), au point qu'une certaine confusion soit possible avec les faciès d'altération mindéliens. C'est sans doute aussi dans cette perspective qu'il convient d'interpréter les profils des arrêts $n^{\circ} 19$ et 23 .

\section{III. - CONCLUSION : LE PROBLEME DES GLACIATIONS PYRENEENNES}

L'étude du piémont garonnais ne peut donner, à elle seule, une vue suffisamment complète de cette question. Néanmoins, en la combinant aux observations portant sur le domaine ariégeois et l'ensemble de la chaîne (P. Barrère 1953, 1963 ; H. Enjalbert 1960 ; J. Hubschman 1975 a ; F. Taillefer 1951, 1969, 1974 ; G. Viers 1963), il est possible d'en dégager les éléments suivants :

- L'existence de deux périodes glaciaires, Riss et Würm, semble aujourd'hui pratiquement indiscutable.

- L'existence de glaciations antérissiennes, mindélienne en particulier, bien que tout à fait envisageable en théorie, reste encore à démontrer pour les partisans d'une attribution au Riss du Maximum glaciaire (type "Glaciaire ancien" de Seilhan évoqué à l'arrêt $n^{\circ} 23$ ). En outre, un certain nombre de faits annexes, qu'il serait imprudent de sous-estimer, ne plaident pas en faveur de ces éventuelles glaciations anciennes. Ils désignent plutôt les remblaiements alluviaux antérissiens comme l'aboutissement d'autant de crises bioclimatiques où la note semi-aride (et probablement froide ou fraîche) semble l'emporter sur l'ambiance franchement froide et humide qu'implique la constitution de puissantes réserves de glaces dans les Pyrénées. On peut rappeler quelques-unes de ces présomptions :

a) Les preuves directes de phases glaciaires antérissiennes font défaut: pas de moraines antérissiennes véritablement probantes (étant entendu que des faciès comme ceux des arrêts $n^{\circ} 19$ et 23 -terrasse de Seilhan - sont alors considérés comme rissiens); impuissance de la morphométrie et de la sédimentologie des nappes antérissiennes à démontrer le caractère indiscutablement fluvio-glaciaire d'alluvions fatalement hétérométriques et très grossières, puisque déposées au pied même de la montagne.

b) Dans les Pyrénées, le Riss semble représenter une rupture paléontologique "froide" majeure. Les associations d'espèces à affinité franchement froide n'apparaissent pratiquement jamais dans les complexes faunistiques antérissiens (L. Méroc 1953, M. Girard, 1973). Au contraire, les faunes "chaudes" héritées du Tertiaire et encore présentes au Mindel-Riss disparaissent totalement au Riss, comme si, depuis la fin du Pliocène, elles s'étaient tant bien que mal adaptées aux crises bioclimatiques jusqu'au moment où, devenues brusquement trop contraignantes, les conditions rissiennes auraient entraîné leur rapide extinction.

c) Certains indices sédimentologiques et géomorphologiques permettent de distinguer plus ou moins clairement les modalités de mise en place des alluvions 
würmiennes et rissiennes, de celles du Donau, Günz et Mindel, surtout à l'aval des Prépyrénées :

. La puissance des alluvions du Würm et du Riss est relativement faible et constante de l'amont vers l'aval, 3 à $6 \mathrm{~m}$ en moyenne ; parallèlement, la taille des galets décroît très progressivement. Le profil transversal des terrasses est très proche de l'horizontalité et leur dessin régulier, fermement limité par les talus Riss-Würm et Mindel-Riss, évoque des organismes fluviaux bien calibrés. Tout ceci traduit sans doute des écoulements régulièrement alimentés et au débit soutenu, en rapport avec l'englacement des vallées pyrénéennes et les climats globalement humides et froids qui prévalaient alors.

Au contraire, la nappe du Donau et, dans une moindre mesure, celles du Günz et du Mindel, montre un rapide amenuisement de la taille des galets de l'amont à l'aval, tandis que l'épaisseur moyenne des alluvions est plus considérable et, pour le Donau, souvent supérieure à 10 et même $15 \mathrm{~m}$, fait déjà noté par E. Harlé en 1895. Enfin, la pente transversale des terrasses est plus marquée, surtout pour le Donau. Ces particularités suggèrent plutôt des écoulements moins régulièrement alimentés et moins aptes à évacuer leur charge au loin que pendant le Riss et le Würm. Elles suggèrent également une mise en place sous forme de larges épandages à chenaux instables et inclinés transversalement. Ce sont là des traits qui pourraient traduire un contexte bioclimatique à tendance semi-aride, plus marqué pour le Donau que pour le Günz et le Mindel. On rejoint ici les observations faites par P. Barrère et G. Viers dans les Pyrénées orientales et sur le versant espagnol: les constructions alluviales immédiatement antérieures aux moraines les plus anciennes (certainement rissiennes) et synchrones des terrasses mindéliennes de l'aval, se présentent souvent comme de véritables glacis, dont on sait que la genèse implique une combinaison bioclimatique à note aride ou semi-aride.

Mais il faut bien reconnaitre que tous les indices qui viennent d'être évoqués, quoique troublants, ne sont pas absolument déterminants. La question du nombre de glaciations dans les Pyrénées reste donc encore ouverte, dès lors que l'on cherche à se garder d'une transposition rigide et sans nuances du schéma polyglacialiste traditionnel alpin.

\section{Bibliographie}

(Voir p. 214). 